\title{
Erratum: Unexpected softness of bilayer graphene and softening of A-A stacked graphene layers [Phys. Rev. B 101, 125421 (2020)]
}

\author{
Y. W. Sun ${ }^{\circ}$, D. Holec, D. Gehringer, O. Fenwick, D. J. Dunstan, and C. J. Humphreys
}

(Received 24 November 2020; revised 12 February 2021; published 12 March 2021)

DOI: 10.1103/PhysRevB.103.119901

There are quantitative errors in Figs. 1 and 2 of our paper, affecting its first conclusion. Stress evaluation employing the Vienna $a b$ initio simulation package (VASP) on inhomogeneous structures and, in particular, on structures where internal strain occurs, requires special handling. Bilayer graphene is such a structure with a large vacuum separation in the supercell along the $c$ axis perpendicular to the graphene plane. We misinterpreted the stress presented in the original Fig. 1 as the stress on the graphene layers. We correct the stress in Fig. 1 below by a simple scaling following the approach in the literature [1,2]. The key message is qualitatively consistent with the original paper, that bilayer graphene stiffens more slowly than graphite over the compression range before the rehybridization of $s p^{2}$ to $s p^{3}$ (i.e., the curve of the stress against the interlayer spacing of the bilayer is shallower than graphite in this range). Additionally, a systematic error in integrating the charge density influenced the discussion of the charge between graphene layers. This error is corrected in Fig. 2, and its impact is discussed in the corresponding text below. Despite the errors arising from these methodological mishandlings, the original interpretation and conclusions remain qualitatively unchanged.

We modeled bilayer graphene by having two layers of graphene in a large supercell with a fixed height $Z=24.65 \AA$ (for a large vacuum separation) along the $c$ axis perpendicular to the graphene layers. The graphene layers were at a variable spacing $d$, and the remainder of the space in the supercell was vacuum. The first derivative of the total energy of the supercell with respect to a displacement $\mathbf{q}$, evaluated at $\mathbf{q}=0$, gives the magnitude of the force $\mathbf{F}$. The type of displacement determines what force we obtain. For example, the displacement of a carbon atom along the $c$ axis yields the force on that atom along this direction; the displacement of the whole graphene plane along the $c$ axis gives the force and, hence, the stress, on that plane. The stress tensor evaluated by VASP by default is the stress on the supercell, evaluated with $\mathbf{q}$ being the deformation of the supercell as a whole, i.e., including its contents. That is, the strain between any two atoms inside the supercell is the same (affine displacements). Unless the system is then relaxed (equilibrated), before the calculation of the change in energy, the results for inhomogeneous systems or systems with internal strain are incorrect.

With our large vacuum separation, we expect the stress $\sigma_{33}$ along the $c$ axis on the supercell to be zero. As it was not zero, we misinterpreted it as the stress on the graphene. Indeed, the energy change was not large as the graphene separation changed by the factor $d / Z$ less than the change in the height of the supercell, where $d$ is the each fixed distance between the layers in bilayer graphene. Interestingly, Mounet and Marzari obtained $c_{11}+c_{12}$ of graphite from the second differential of energy with respect to biaxial strain, but they did not get $c_{11}$ or $c_{12}$ in this way as the required strains cause internal strain in the four-atom unit cell [3]. Instead, they obtained them from phonon dispersions. Liu et al. also explicitly state the need for the graphene structure to be relaxed after every deformation before calculating Young's modulus and Poisson's ratio [1]. On the other hand, they did use the stress on the supercell containing graphene and vacuum, which they referred to as a stress averaged over the supercell volume. They, therefore, corrected it by the factor $Z / d_{0}$ for calculating the stress on the graphene from the supercell stress. Capaz et al. used a similar approach to evaluate the stress along a carbon nanotube axis after straining the tube along this direction by a scaling factor of the length of the unit cell in the dimension with a large vacuum separation (to model an individual tube) over the diameter of the tube cross section [2]. Although the bilayer graphene is under internal strain (except at equilibrium), the stress on the layers can be directly evaluated by VASP (from affine displacements) with a scaling factor because the contribution of a small deformation of the large vacuum (height over $20 \AA$ ) to the total energy is negligible. In Fig. 1, we present the stress corrected by multiplying by the factor $Z / d$.

The corrected figure shows that for both Bernal and A-A stackings, to compress to a smaller interlayer distance, the required uniaxial stress along the $c$ axis increases more slowly on a bilayer graphene than graphite. This key conclusion is consistent with the original paper.

The error in the original Fig. 2 is more subtle: The integrated charge density suffered from numerical issues. The charge density is calculated on a grid, sampling the whole simulation cell, i.e., it is known only as a discrete quantity. The chosen step for sampling the bilayer interlayer distance (of $0.1 \AA$ ) was not commensurate with the simulation box dimension (of $24.65 \AA$ ) and grid size (of 500 points). Therefore, whereas one graphene layer (at $z=0$ ) always remained at a charge-density grid point, the other layer "moved" to different relative positions between two grid points. As our fast Fourier transform (FFT) grid spacing in the $z$ direction is $\Delta c_{\mathrm{FFT}}=0.0493 \AA$, moving a plane over a distance of $0.1 \AA$ means moving it by two FFT grid points 


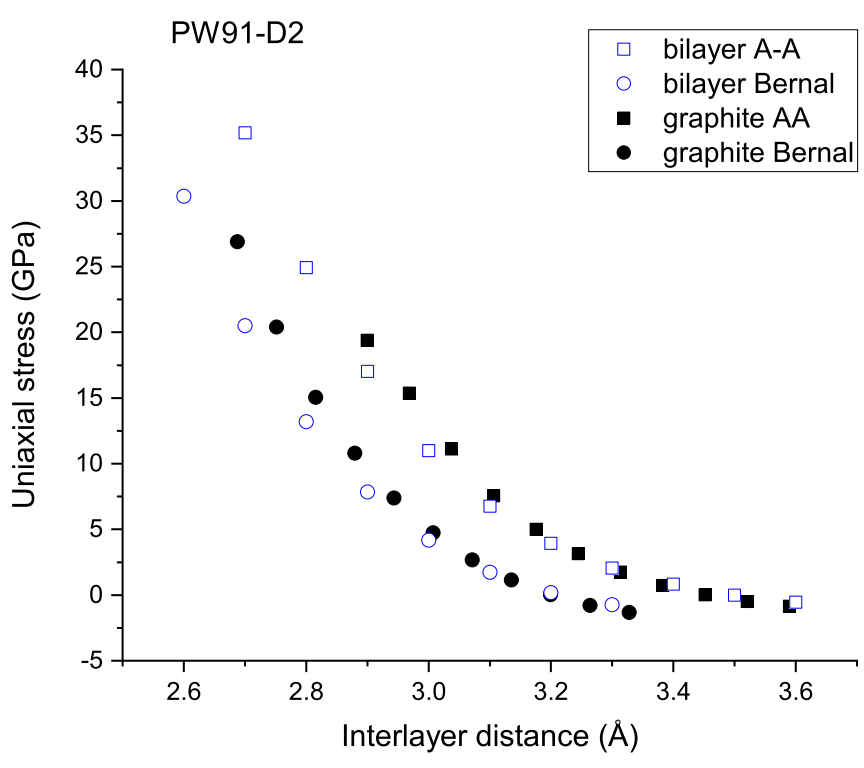

FIG. 1. Corrected Fig. 1 by simply multiplying a scaling factor. The uniaxial stress along the $c$ axis applied to A-A and Bernal-stacked bilayer graphene and graphite is plotted with the corresponding interlayer distance at which the stress was calculated. The black solid points are for graphite, and the blue open circles are for bilayer graphene. The circles are for Bernal stacking, and the squares are for A-A.

plus an offset $\Delta c_{\text {off }}=\left(0.1-2 \Delta c_{\mathrm{FFT}}\right) / \Delta c_{\mathrm{FFT}}$ which calculates to be $\approx 2.8 \%$ of $\Delta c_{\text {off }}$. Consequently, the (numerically) integrated charges suffered from systematic errors since the values of $\rho$ [within the projector augmented-wave spheres (near-core)] on the FFT grid are sensitive to their distance from the atomic centers, resulting in largely overestimated charge-density changes. We now fix the relative position of the graphene layer to the grid points. The corrected va-

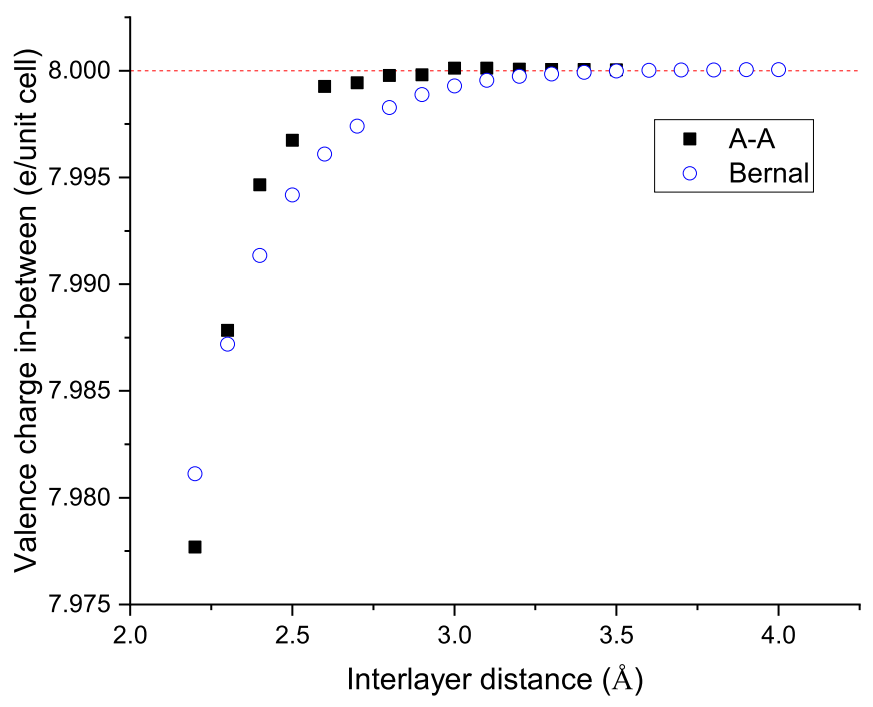

FIG. 2. Corrected Fig. 2. The integrated valence charge between the two graphene layers is plotted versus the interlayer distance of bilayer graphene for A-A (blue open circles) and Bernal stackings (black solid squares). The horizontal dashed line is for graphite.

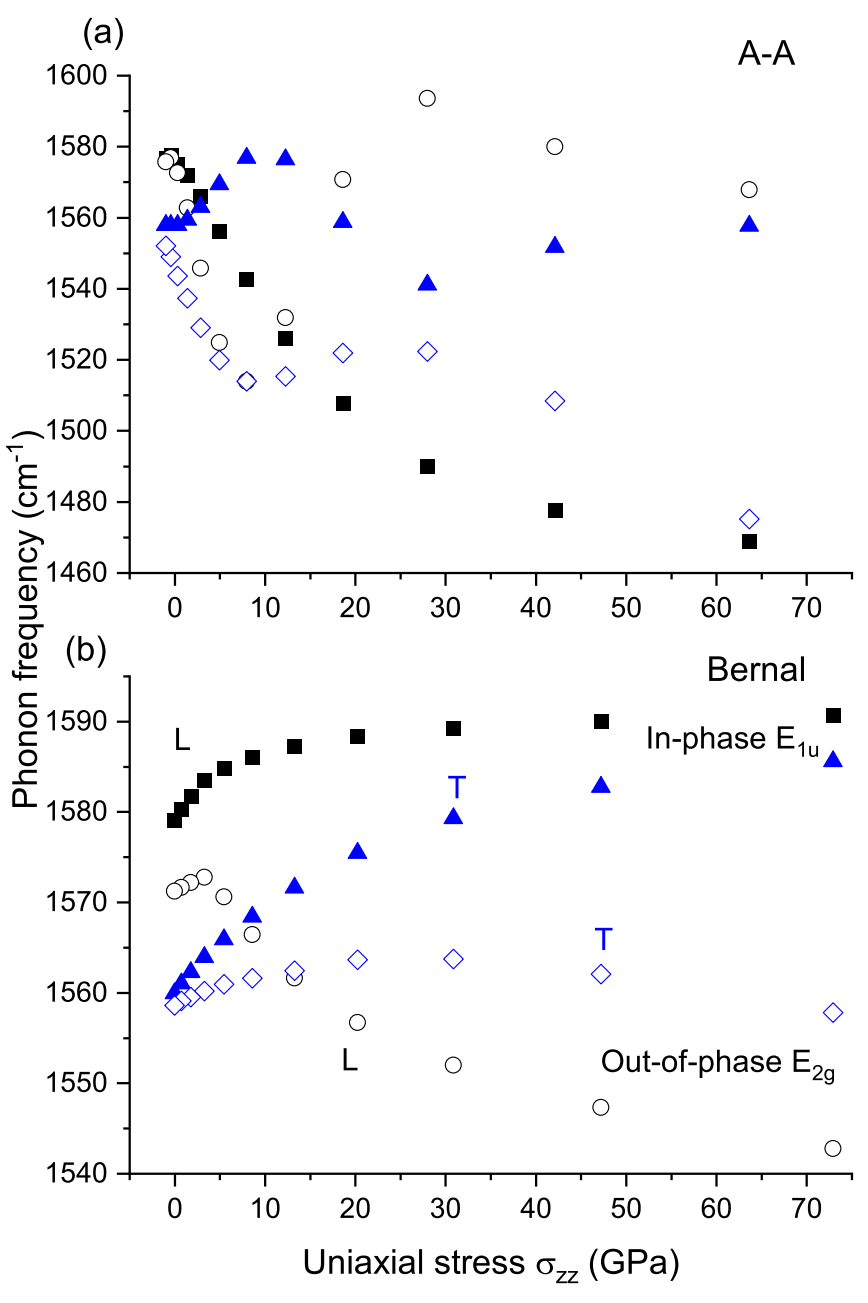

FIG. 3. Corrected Fig. 1 in the SI. The caption remains the same: The frequencies of the four in-plane phonons of (a) A-A- and (b) Bernal-stacked bilayer graphene are plotted with uniaxial stress along the $c$ axis. The solid points are for the two-plane in-phase modes $\left(E_{1 u}\right)$, and the open points are for the two-plane out-of-phase modes $\left(E_{2 g}\right)$. The black points are for the modes along the longitudinal direction on the hexagonal plane of graphene $(L)$, and the blue points are for the modes along the transverse direction $(T)$.

lence charge between graphene layers at various interlayer distances is presented in Fig. 2, replacing the old Fig. 2. Electrons are squeezed through graphene planes for both Bernal- and A-A-stacked bilayer graphene where the original interpretation applies: This unexpected softness is related to the possibility of electrons being squeezed through graphene planes.

Due to the same error in Fig. 1, Fig. 1 in the Supplemental Material (SI) is corrected and replaced by Fig. 3 with the corrected values of the stress. The message is unchanged that in-plane phonon frequencies shift nonmonotonically in an A-A stacked bilayer, suggesting a large disruption of the $s p^{2}$-orbital distribution under out-of-plane compression, in addition to electrons being squeezed through the graphene plane discussed above. A further correction is that in the caption of Fig. 5, bilayer graphene should be replaced by graphite. 


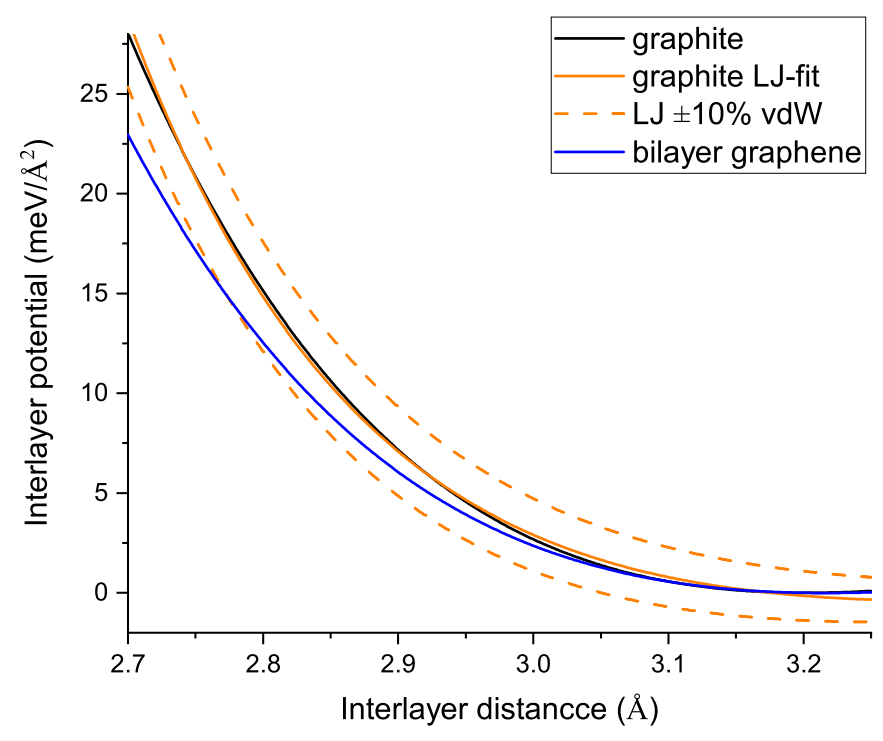

FIG. 4. The interlayer potential of Bernal-stacked graphite and bilayer graphene is plotted with interlayer distance. The black line is the data for graphite. The orange solid line is the fit using the Lennard-Jones (LJ) potential (almost overlapping with the data). The orange dashed lines are modified LJ potentials with a $10 \%$ increased or decreased attraction coefficient, respectively, whereas the repulsion is the same as the orange solid line. The blue line is the data for bilayer graphene.

In conclusion, we have corrected the results of the first part of the original paper. The key message that bilayer graphene stiffens more slowly than graphite along the $c$ axis perpendicular to the graphene plane (i.e., the stress increases more slowly with decreasing interlayer spacing in the bilayer) is unchanged. The quantitative description has been corrected. The proposed mechanism, that this softness is related to the possibility of electrons being squeezed through graphene layers, is also unchanged.

Figure 4 demonstrates that the choice of van der Waals (vdW) corrections does not affect our key message that bilayer graphene stiffens more slowly than graphite. We interpolate the data of Bernal-stacked graphite in Fig. 1 (black circles). We obtain the interlayer potential at an interlayer distance by integrating uniaxial stress along the $c$ axis over the interlayer distance from the calculated distance at equilibrium to this distance. We use the LJ potential in the A-B form with a vertical offset to make the energy minimum at zero [4]: $V_{L J}(r)=\frac{A}{r^{12}}-\frac{B}{r^{6}}+C$ to fit the interlayer potential. The fit (orange solid line) almost overlaps with the data (black solid lines). We then plot the modified LJ potential by increasing or decreasing the attraction coefficient $B$ by $10 \%$, whereas fixing the repulsion coefficient $A$ and offset $C$ at the fitted values (two orange dashed lines, respectively, to the increased and decreased $B$ ). We compare the interlayer potential of graphite with its attraction term modified to that of bilayer graphene.

The message is clear, that the attraction term affects the energy minimum and the interlayer distance at equilibrium but not the curvature of the interlayer potential-distance relation, and, therefore, the choice of vdW corrections cannot be responsible for the separation between graphite and bilayer graphene in the compressibility behavior shown here.

We do not intend to argue which correction is superior to another, in better describing the vdW, determining the interlayer spacing at equilibrium because equilibrium position is not a concern of this erratum. Therefore, the comparison of the elastic constant $c_{33}$ at equilibrium between graphite and bilayer graphene in the original paper was misleading. The relevant parameter is the variation of the out-of-plane stiffness, the curvature of the interlayer stress-distance relation, presented in Fig. 1.
[1] F. Liu, P. Ming, and J. Li, Ab initio calculation of ideal strength and phonon instability of graphene under tension, Phys. Rev. B 76, 064120 (2007).

[2] R. B. Capaz, C. D. Spataru, P. Tangney, M. L. Cohen, and S. G. Louie, Hydrostatic pressure effects on the structural and electronic properties of carbon nanotubes, Phys. Status Solidi B 241, 3352 (2004).
[3] N. Mounet and N. Marzari, First-principles determination of the structural, vibrational and thermodynamic properties of diamond, graphite, and derivatives, Phys. Rev. B 71, 205214 (2005).

[4] J. E. Lennard-Jones, Cohesion, Proc. Phys. Soc., London Sect. A 43, 461 (1931). 